Rodríguez Bustamante, A., Vicuña Romero, J. J., y Zapata Posada, J. J. (mayo-agosto, 2021).

Familia y escuela: educación afectivo-sexual en las escuelas de familia. Revista Virtual

Universidad Católica del Norte, (63), 312-344. https://www.doi.org/10.35575/rvucn.n63a12

\title{
Familia y escuela: educación afectivo-sexual en las escuelas de familia
}

Family and school: affective-sexual education in family schools

\author{
Alexander Rodríguez Bustamante \\ Doctorando en Educación \\ Escuela de Educación y Pedagogía, Universidad Pontifica Bolivariana \\ Director Escuela de Posgrados. Universidad Católica Luis Amigó. \\ Medellín, Colombia \\ alexander.rodriguez@upb.edu.co \\ Orcid: https://orcid.org/0000-0001-6478-1414 \\ CvLac: https://scienti.minciencias.gov.co/cvlac/visualizador/generarCurriculoCv.do?cod_rh=0001325256
}

\section{Jenny J. Vicuña Romero}

Ph.D. en Ciencias de la Educación

Escuela de Educación y Pedagogía, Universidad Pontifica Bolivariana

Medellín, Colombia

jenny.vicuna@upb.edu.co

Orcid: Orcid: https://orcid.org/0000-0002-8914-9535

CvLac:

http://scienti.colciencias.gov.co:8081/cvlac/visualizador/generarCurriculoCv.do?cod rh=0001609913

\section{Johanna J. Zapata Posada}

Ph.D. en Desarrollo y Ciudadanía: Derechos Humanos, Igualdad, Educación e Intervención Social

Escuela de Ciencias Sociales, Universidad Pontificia Bolivariana

johanna.zapata@upb.edu.co

Orcid: https://orcid.org/0000-0003-3897-0451

CvLac:

http://scienti.colciencias.gov.co:8081/cvlac/visualizador/generarCurriculoCv.do?cod_rh=0000678490

Recibido: 21 de octubre de 2020

Evaluado: 4 de diciembre de 2020

Aprobado: 26 de febrero de 2021

Tipo de artículo: Revisión 


\title{
Resumen
}

Develar la relación escuela-familia como vínculo portencializador de la educación afectivosexual. Para la indagación, se utilizaron descriptores como: familia y escuela, escuelas de padres, escuelas de familia, educación sexual y familia, educación sexual y escuela, educación sexual y perspectiva de género; posteriormente, se seleccionaron las referencias con artículos que cumplían los criterios de inclusión en un margen de tres décadas (1991-2020), que recoge 61 documentos para consolidar el estado del arte. El análisis permitió construir una matriz bibliográfica, mapas sinópticos y de análisis conceptual que se ordenaron lógicamente en 4 categorías: Lo familiar en la sociedad, Escuela: territorio para el encuentro, Escuelas de Familia, y Educación afectivo-sexual: entre lo público y lo privado. La relación familia-escuela es un tema de actualidad, donde los roles y funciones de ambas instituciones se complejizan en escenarios que retan las estructuras tradicionales en la educación. El abordaje situado a la realidad actual y nacional muestra una propuesta necesaria y pertinente, desde el enfoque de derechos humanos, para el fortalecimiento de la relación familia-escuela, a pesar de los retos a los que se enfrenta la escuela en la actualidad (diversidad en tipologías de familia, contextos, roles, estereotipos del género, entre otros).

Palabras clave: Afectividad; Educación sexual; Escuela; Escuelas de familia; Familia.

\begin{abstract}
Objective. Unveil the school-family relationship as a potentializing link in affective-sexual education. Methodology. For the investigation, descriptors were used such as: family and school, parent's schools, family schools, sexual education and family, sexual education and school, sexual education and gender perspective; subsequently, the references with articles that met the inclusion criteria were selected in a margin of three decades (1991-2020) that collects 61 documents to consolidate the state of the art. The analysis allows the construction of a bibliographic matrix, synoptic and conceptual analysis maps that are logically ordered into 4 categories: The familiar in society, School: territory for the encounter, Family Schools; and Affective-sexual education: between the public and the private. Results. The family-school
\end{abstract}


relationship is a current issue, where the roles and functions of both institutions become more complex in scenarios that challenge traditional structures in education. Conclusion. The approach situated to the current and national reality shows a necessary and pertinent proposal from the Human Rights perspective for the strengthening of the family-school relationship despite the challenges that the school is currently facing (diversity in types of family, contexts, roles and gender stereotypes; among others).

Keywords: Affectivity; Sex education; School; Family Schools, Family.

\section{Introducción}

En cuanto necesidad ontológica, la esperanza necesita de la práctica para volverse historia concreta. Por eso no hay esperanza en la pura espera, ni tampoco se alcanza lo que se espera en la espera pura, que así se vuelve espera vana. (Freire, 2002, p. 8)

El escrito que se presenta a continuación es resultado de la construcción de la propuesta de trabajo doctoral denominado: "Familia y escuela: educación afectivo-sexual en las escuelas de familia”, adelantada en el Doctorado en Educación de la Universidad Pontificia Bolivariana UPB-, la cual centra su interés en la participación de los diferentes actores del medio escolar en las escuelas de familia; ejercicio participativo transformador en las instituciones educativas y a su vez un medio potencial para la educación afectivo-sexual. Este binomio parte de la idea que la relación familia y escuela representa un tema crucial y relevante para las sociedades actuales, en donde los roles y funciones, de una institución y de otra, se complejizan en escenarios que retan las estructuras educativas tradicionales.

Este reto que presupone el interrogar la familia y la escuela como espacios esenciales en la socialización de hombres y mujeres y, en especial, de lo afectivo-sexual en lo educativo, es resultado de los cambios que, tanto a nivel institucional como social, se han presentado como resultado del posicionamiento de discursos alternativos e inclusivos generados desde la académica, frente a la responsabilidad compartida en la formación de quienes hacen parte del sistema educativo.

Ineludiblemente la escuela, como escenario educativo, no pudo abstraerse de los cambios 
socio históricos e ideológicos emergentes; lo que tuvo como principal consecuencia la sobreinstitucionalización del cuidado de niños y niñas (Issó-García, 2012), donde la función del docente trasciende de sujeto transmisor de conocimientos formales a instaurar relaciones afectivas con los estudiantes, para formar en aspectos como la autonomía y la responsabilidad (Ochoa-Ninapaitán, 2018). Ello va a dar respuesta a los cambios acaecidos en el mundo familiar a partir de la salida de la mujer al mercado laboral y de la aparición de actores públicos y privados que van a participar en actividades de cuidado y educación, que antes solo realizaban las mujeres; es decir, se rompe de esta manera con la división sexual del trabajo, donde la proveeduría económica se le atribuía a la figura del padre y el sostenimiento emocional a la madre (Montoya-Ahmedt, 2017); soporte socio político evidenciado en los lineamientos normativos heredados, como la Ley 294 de 1996, y las concepciones familistas (Puyana, 2007) que allí operan.

Es así como las tareas originalmente atribuibles a las unidades familiares son compartidas, en la actualidad, con las instituciones de educación formal; puede decirse que "parental involvement in children's learning has long been advocated as the key to their success and to school improvement" [la participación de los padres en el aprendizaje de los niños ha sido defendida durante mucho tiempo como la clave para su éxito y para la mejora escolar] (Wee, 2011, p. 48). Dicho de otro modo:

Tanto la escuela como la familia siguen siendo los entornos más próximos y también los más adecuados para el desarrollo del ser humano; estos ámbitos deberán entonces flexibilizarse, abrirse y apoyarse mutuamente. Por lo que la participación de los padres es una condición necesaria para facilitar el desarrollo de las nuevas generaciones, propósito que ambas instituciones (familia y escuela) persiguen. (Márquez-Ibarra, 2014, p. 52)

Así mismo, la participación de la familia en los procesos educativos de los niños y niñas se encuentra legalmente establecida en las normativas colombianas. Es el caso de la Ley 1098 de 2006 que, en su artículo décimo, enuncia la concurrencia de actores sociales para la protección de los derechos, y en su artículo 14 establece la responsabilidad parental como medio para alcanzar el máximo de satisfacción de los derechos y formación integral de niños y niñas. De igual modo, en la Ley 1404 de 2010 se disponía como objetivo "integrar a todos los padres y madres de familia, así como a los acudientes a un cuerpo organizado que se articule con la 
comunidad educativa, principalmente docentes, alumnos y directivos" (párr. 1).

Más recientemente, para propiciar y garantizar la participación de las familias en los espacios educativos, se promulga la Ley 2025 de 23 de julio del 2020, por medio de la cual se establecen lineamientos para la implementación de las escuelas para padres, madres de familia y cuidadores, en las instituciones de educación preescolar, básica y media del país, derogando la Ley 1404 de 2010. Cabe señalar que su implementación tiene carácter de obligatoriedad en todas las instituciones educativas del país y debe estar articulada a los proyectos educativos institucionales.

En consecuencia, a la institución familiar le corresponde participar en actividades vinculadas a la escuela, con miras a la formación integral: académica, social, de valores y principios, de los estudiantes de preescolar, básica y media en las instituciones educativas públicas y privadas. Estos lineamientos propuestos evidencian la intencionalidad de protección y reconocimiento de los niños y niñas como sujetos de derechos, únicos, dignos y capaces de influir y de dejarse influir por el contexto.

En este sentido, se reconoce que interrogar el mundo familiar desde el escenario educativo, como es la escuela, permite comprender el contexto multidimensional al que se enfrenta el docente, como los actores institucionales, en cuanto a la posibilidad de ofrecer un escenario en el cual se reconozcan las historias, prácticas, costumbres y creencias de los niños y niñas y sus familias, así como las capacidades y emociones que configuran para dar respuesta a las necesidades y desafíos que impone este medio. Es por ende necesario ver el proceso de enseñanza-aprendizaje como una acción integral que recoge al niño en clave de su desarrollo humano integral.

El desarrollo humano integral como categoría analítica es amplia, por ello surge la necesidad de enfocar el análisis en un indicador de los múltiples que se podrían tener en cuenta, en este caso la educación afectivo-sexual. Esta se concibe como proceso de construcción de explicaciones, valores, normas y creencias, en relación con la sexualidad humana, en la que se ha de tener en cuenta las potencialidades y la libertad de las familias, educandos y educadores (Barragán-Medero, 1991). Esta apuesta que invita a no pensar lo público y lo privado como espacios dicotómicos, sino como esferas complejas e interrelacionadas que convocan a generar nuevas comprensiones, en cuanto a que allí la cultura va a definir la interacción afectiva y 
cognitiva entre los individuos y, en especial, de los niños y niñas, permite justificar el porqué de la necesidad del develar la relación escuela-familia en clave de ser potenciadores de la educación afectivo-sexual.

Para ello, se requiere de la (re)construcción de canales de comunicación, como las escuelas de familias, que permitan reenfocar el objetivo de este proceso educativo: contribuir al desarrollo integral de seres humanos; lo que trae consigo el reconocimiento implícito de las funciones propias e intransferibles de cada institución. y precisa de continuidad desde la formalidad propia del contexto escolar o desde las pautas educativas espontáneas que se consolidan en las configuraciones familiares.

En este sentido, Márquez-Ibarra (2014) refiere que la escuela se dispone al encuentro de las familias en un escenario político de deberes frente a la formación no solo de los estudiantes, sino de sujetos políticos en una organización estructurada, hacia la formación de nuevas generaciones, donde el aporte docente en las instituciones no es el único que se requiere para tal fin; en conjunto con los padres, madres y otros representantes de las familias, se genera el reconocimiento y la integración del contexto.

Finalmente, para cerrar este apartado se debe señalar que la corresponsabilidad entre escuela y familia en la formación de los niños y niñas se constituye en un deber y un derecho, puesto que el diálogo trasciende a la interconexión de narrativas, en donde la heterogeneidad de lenguajes, experiencias y formas de ser y hacer familias componen una red de sentido comunitario (Dabas et al., 2011) y fortalecen relaciones basadas en el acompañamiento y la compresión, de manera que la articulación de las acciones de la familia y la escuela generen un sentido de participación y corresponsabilidad (Galián et al., 2018).

\section{Método}

Este abordaje se realizó bajo el enfoque de investigación cualitativo; de acuerdo con Guerrero-Bejarano (2016) a través de este se pretende comprender una realidad social configurada por las interacciones entre los sujetos y su entorno, a través de la descripción, análisis, interpretación y develamiento de las cualidades del fenómeno en estudio. A partir del objetivo propuesto en este texto, en cuanto al develar la relación escuela-familia como vínculo 
potencializador de la educación afectivo-sexual, se optó porque este enfoque se transversalizara por la estrategia del estado del arte, entendido este como:

El estado del arte es una investigación sobre la producción investigativa de un determinado fenómeno. Este permite develar la dinámica a partir de la cual se ha desarrollado la descripción, explicación o comprensión del fenómeno en estudio y la construcción de conocimientos sobre el saber acumulado. Es, también, un elemento básico e indispensable para definir y estructurar la investigación. (Guevara-Patiño, 2016, p. 169)

Para el análisis de las voces de los autores, incluidos en el presente texto, se recurrió a la hermenéutica. A través de esta se logró develar los sentidos interpretativos que los autores revisados entregaban de la realidad objeto de estudio, sin olvidar que allí también se cuestiona, confronta y agregan otras voces que están en sintonía con la temática propiamente o derivadas de estas (Damiani, 2020). Es por ello por lo que, como señalan Denzin y Lincol (2012), la aplicación de la hermenéutica al estado del arte permite realizar un ejercicio interpretativo desde lo históricosocial de los relatos de los sujetos de investigación, a través del cual se logra una reconstrucción textual de manera bidireccional con el investigador.

Para la construcción del corpus sujeto de análisis e interpretación, se desarrolló en un primer momento la búsqueda documental, a través de los siguientes descriptores, que recoge un sistema de categorías pensadas a la luz de la relación escuela-familia y educación afectivo-sexual: familia y escuela, escuelas de padres, escuelas de familia, educación sexual y familia, educación sexual y escuela, educación sexual y perspectiva de género. Como técnica de recolección de información se tuvo en cuenta el análisis de dominio.

En un segundo momento, a partir del rastreo realizado en las bases de datos de los descriptores anteriormente señalados, se establecieron los siguientes criterios de inclusión: artículos publicados en revistas científicas; tesis de maestría y doctorado ubicadas en repositorios institucionales nacionales o internacionales; el año de publicación se ubica entre el periodo19912020; finalmente, que el énfasis fuera en el área de educación. Para el desarrollo de este texto se revisaron 61 textos, entre los que se encuentran 13 tesis de doctorado, 5 tesis de maestría, 1 trabajo de pregrado, 34 artículos académicos, 6 leyes en el marco normativo colombiano, 1 documento público, 7 libros, y 1 página web oficial del Fondo de Población de las Naciones Unidas. Es importante aclarar que cuando esto se llevó al siguiente momento de análisis categorial, solo se 
hicieron evidente 54 referencias bibliográficas (ver tabla 1), que recogen los planteamientos primarios, a través de los cuales los autores de este artículo desarrollan sus discusiones frente a cada una de las categorías construidas.

En un último momento, a partir de los textos seleccionados, se construyó una matriz bibliográfica, con el fin de dilucidar datos como: tipo de material, autor, año, título, categoría y locación del texto. Asimismo, se recurrió al uso de mapas sinópticos y de análisis conceptual para dar un orden lógico al conjunto de categorías y unidades temáticas, lo que permitió la definición de cuatro categorías para el desarrollo de los resultados: (1) Lo familiar en la sociedad, (2) Escuela: territorio para el encuentro, (3) Escuelas de familia; y (4) Educación afectivo-sexual: entre lo público y lo privado; cuyo contenido temático da respuesta al objetivo planteado como horizonte escritural de este trabajo.

\section{Tabla 1}

\section{Distribución bibliográfica categorial}

\begin{tabular}{cll}
\hline \multicolumn{1}{c}{ Categorías } & \multicolumn{1}{c}{ Referencias } \\
\hline 1. La familia en la sociedad & $\begin{array}{l}\text { 11 textos: artículos académicos, leyes, libros y tesis } \\
\text { doctorales. }\end{array}$ \\
\hline 2. Escuela: territorio para el encuentro & $\begin{array}{l}12 \text { textos: artículos académicos de investigación, } \\
\text { libros, leyes, tesis doctorales y trabajos de maestría. }\end{array}$ \\
\hline 3. Escuelas familiares & $\begin{array}{l}13 \text { textos: artículos académicos, leyes, trabajos de } \\
\text { maestría y tesis doctorales. }\end{array}$ \\
\hline 4. Educación afectivo-sexual: entre lo & $\begin{array}{l}18 \text { textos: Artículos, libros de páginas web, un trabajo } \\
\text { de grado, trabajos de maestría y tesis de doctorado. }\end{array}$ \\
\hline
\end{tabular}

Nota: Elaborado por Rodríguez Bustamante Alexander.

\section{Resultados}

A continuación, se presentarán los principales hallazgos resultado del arqueo documental, cuya estructura argumentativa está fundamentada en las cuatro categorías resultado de la clasificación del acervo bibliografía, incluido y relacionado en el acápite anterior. Las categorías son: (1) Lo familiar en la sociedad, (2) Escuela: territorio para el encuentro, (3) Escuelas de Familia, y (4) Educación afectivo-sexual: entre lo público y lo privado. Es importante señalar que en este apartado se logran identificar líneas argumentativas de encuentro entre autores, contextos y 
lecturas críticas, pero también hay espacio para visibilizar las fisuras entre estos a la hora de abordar escuela-familia y educación afectivo-sexual en contextos educativos.

\section{Lo familiar en la sociedad}

Para ampliar la comprensión de la institución familia en la sociedad, es necesario, en un primer momento, abordar el concepto de familia; sin embargo, las conceptualizaciones son diversas, debatibles, y hasta contradictorias, lo que contribuye a que el tema de familia se torne escurridizo, y corre el riesgo de ser invisibilizado o reducido a situaciones problemáticas de la sociedad que involucran a sus integrantes (delincuencia, consumo de sustancias psicoactivas, maternidad y paternidad juvenil, entre otras).

Es por ello importante señalar la dificultad de definir familia, ya que su carácter cambiante, resultado de los individuos que la integran y de su relación con el mundo social, donde confluyen asuntos sociales, políticos, económicos, culturales, tecnológicos e ideológicos, han traído consecuencias que la han modificado en su estructura, dinámica y organización interna. Esta nueva realidad invita a continuar deconstruyéndola, para de esta manera identificar sus cambios en sí misma y en la relación con la realidad social, eje fundamental desde donde se piensa la actuación profesional, en especial la escuela, quien además cumple una tarea de ser agente socializador de las nuevas generaciones.

En este sentido, Carvajal et al. (2018) van a señalar la relación entre familia y escuela a partir del papel que juegan ambas en el desarrollo humano y afectivo del niño y la niña; es decir, una tarea de cuidado en su formación como sujeto partícipe en la sociedad, lo cual va a significar un vínculo de responsabilidad compartida a cumplir,

La familia ha representado para el niño el agente educativo de mayor valor afectivo y su primera escuela. El trabajo educativo que en ella se desarrolle sienta las bases para la formación de su personalidad y en correspondencia, es una necesidad que los miembros sepan cuidarlos, alimentarlos, educarlos, quererlos y amarlos. (p. 33)

Por su parte, Ros-Codoñer (2018) va a profundizar en este vínculo al afirmar que uno de los ejes diferenciales de la producción relacional de la familia, es la capacidad de redistribución del poder entre los integrantes, de acuerdo a sus características e independientemente si hay 
limitaciones individuales o colectivas; de esta manera, se reconoce que la familia puede desarrollar posibilidades en la cotidianidad para el cuidado y acompañamiento de hijos, cónyuges, abuelos, hermanos u otras personas en situaciones desfavorables o de vulnerabilidad. Por tanto, en estas situaciones anteriormente mencionadas, la labor educativa de la familia esta ineludiblemente enlazada con "el hecho de mantener una relación más democrática, basada en el diálogo y la confianza" (Esteban, 2015, p. 74). Ello posibilita la creación de escenarios para el desarrollo de capacidades, permitiendo la producción de capital social y humano que, para RosCodoñer (2018), representa el material primario de la construcción de tejido con sentido para el desarrollo de acciones colectivas que posibiliten el cambio social. En otras palabras "la familia no es solamente el lugar social específicamente humano de la procreación y la intimidad, sino que es la esfera generadora de humanidad para el conjunto societario" (Ros-Codoñer, 2018, p. 224). De este modo, se reconoce que las familias están constituidas por sujetos que comparten historias, prácticas y discursos donde se posibilita la movilización de recursos, capacidades y emociones entre sus integrantes y el medio externo al familiar (Lacasa, 1997).

En síntesis, y retomando a Cano-Muñoz (2015), lo familiar en la sociedad va a entenderse desde la heterogeneidad de familias y de relacionamiento con la escuela; no obstante, estas tienen algunas características afectivas transversales a sus formas de configuración, tales como el compromiso con el desarrollo de quienes la conforman, así como el apoyo recíproco e interdependiente con otras esferas contextuales, en aras de propiciar el desarrollo social. Es decir, el lugar de la familia se debe al cumplimiento de tareas más allá de las que pueden abarcar otros escenarios socializadores para el ser humano, como es la escuela.

\section{Escuela: territorio para el encuentro}

La escuela en su ejercicio de pensarse como institución se ha convertido en objeto y sujeto de múltiples transformaciones a lo largo de la historia de la humanidad, al igual que la institución de la familia (Martínez-Pérez, 2012). En cuanto a las concepciones que se tienen sobre la escuela, se logran identificar dos perspectivas desde este rastreo documental: en primer lugar, se puede ubicar algunos autores como Fúnez (2014), Capera y Torres (2014) y Aguerri (2017), quienes para vislumbrar la conceptualización y funciones de esta institución, parten de la 
característica de complementariedad de los procesos formativos en las unidades familiares como valores, ideales e identidad familiar; no obstante, esta concepción de la escuela deja un aspecto a considerar, y es la interrogante que emerge frente los estudiantes con familias caracterizadas por estilos parentales negligentes, en donde la continuidad formativa se ve seriamente afectada, debido al contacto del centro educativo con el grupo familiar, y viceversa.

Una segunda perspectiva se refiere al concepto de escuela desde la trascendencia de la enseñanza de contenidos informativos hacia aspectos formativos del ser humano en valores, salud integral y sentido comunitario; es precisamente en esta perspectiva en donde hablar de una educación afectivo-sexual integral es pertinente. Autores como Velázquez-Barragán (2010), Martínez-Pérez (2012), Gallego (2016) y Durán de León (2018), coinciden en la necesidad de fortalecer el cerco que puede surgir en el estatus conversacional de los padres y madres de las familias, adultos significativos y la institucionalidad-escuela como una tarea pendiente por discutir y desarrollar. Para mayor claridad se dispone del siguiente tamizaje conceptual (ver tabla 2).

Tabla 2

Tamizaje conceptual lugares comunes familia-escuela

\begin{tabular}{|c|c|}
\hline Autor & Definición \\
\hline $\begin{array}{l}\text { Velázquez- } \\
\text { Barragán } \\
\text { (2010) }\end{array}$ & $\begin{array}{l}\text { Espacio en donde todos los niños y niñas, familias, profesores y comunidad, } \\
\text { independientemente de sus condiciones, pueden conseguir altos niveles de logro; tener } \\
\text { éxito, ser competentes personal y socialmente, participar, aprender dialogando a } \\
\text { convivir y, sentirse que forman una parte importante e insustituible de su entorno social } \\
\text { de referencia (p. 23). }\end{array}$ \\
\hline $\begin{array}{c}\text { Martínez-Pérez } \\
\text { (2012) }\end{array}$ & $\begin{array}{l}\text { Un lugar que potencia las capacidades de cada sujeto y la ayuda a desarrollarse como } \\
\text { personas en todas sus facetas y estableciendo relaciones con los otras (p. 59). }\end{array}$ \\
\hline $\begin{array}{l}\text { Gallego } \\
(2016)\end{array}$ & $\begin{array}{l}\text { (...) debe ayudar al desarrollo de las capacidades, competencias o cualidades humanas } \\
\text { fundamentales que requiere el ciudadano contemporáneo para vivir de manera } \\
\text { satisfactoria (p. 59). }\end{array}$ \\
\hline $\begin{array}{l}\text { Durán de León } \\
\text { (2018) }\end{array}$ & $\begin{array}{l}\text { La escuela, representa el espacio social por excelencia, cuya misión queda claramente } \\
\text { establecida en la formación de un ciudadano republicano, que con reconocimiento de } \\
\text { los valores autónomos se enmarque en un mundo globalizado, en donde el desarrollo } \\
\text { integral de los educandos se exprese en contenidos pedagógicos que consideren el } \\
\text { desarrollo intelectual, la formación del trabajo, la salud física, mental y espiritual, } \\
\text { siempre en la búsqueda del bien común en la colectividad (p. 27). }\end{array}$ \\
\hline
\end{tabular}




\begin{tabular}{|c|c|}
\hline $\begin{array}{l}\text { Rivera } \\
\text { Sepúlveda, Á. } \\
\text { A., y Guerra } \\
\text { García, Y. M. } \\
\quad(2020)\end{array}$ & $\begin{array}{l}\text { Es así como la escuela se ha convertido en un escenario de conflictos y rivalidades } \\
\text { entre grupos con intereses en colisión, que no sólo está erosionando las relaciones entre } \\
\text { la escuela y la comunidad, sino que ha contribuido a reproducir la fragmentación social } \\
\text { y a recrudecer las enemistades entre familiares y vecinos, lo que Machado (2017) } \\
\text { describe como un fenómeno idiosincrático de los sectores rurales en Colombia. (p. } \\
\text { 110). }\end{array}$ \\
\hline $\begin{array}{l}\text { Horna-Clavo, } \\
\text { E., Arhuis- } \\
\text { Inca, W., y } \\
\text { Bazalar- } \\
\text { Palacios, J. } \\
\text { (2020) } \\
\end{array}$ & $\begin{array}{l}\text { El ambiente familiar es necesario para el proceso infantil, y a la vez es protagonista } \\
\text { esencial en la educación de los niños. Las actitudes cotidianas se convierten en } \\
\text { prácticas formativas; actitudes que serán reflejadas por el infante en sus dimensiones } \\
\text { biológica, psicológica y social. (p. 226). }\end{array}$ \\
\hline $\begin{array}{l}\text { Barrera, D. y } \\
\text { Ortiz, M. } \\
\quad(2017)\end{array}$ & $\begin{array}{l}\text { Dentro de la fenomenología relacional del adolescente se encuentran los cambios en los } \\
\text { vínculos familiares, que se manifiestan como un alejamiento de este respecto de sus } \\
\text { padres, en búsqueda de una nueva autonomía; la vivencia grupal, que se articula a la } \\
\text { búsqueda de seguridad y de reconocimiento social; las relaciones de pares que } \\
\text { adquieren un carácter distinto al de la infancia; y la sexualidad y nuevas elecciones } \\
\text { afectivas. (p. 243). }\end{array}$ \\
\hline
\end{tabular}

Nota: elaboración propia.

Estas conceptualizaciones permiten identificar una concepción de la escuela desde la interrelación socializadora con la familia, más allá de pensarse como un continuo educativo, ya sea en las instalaciones educativas o en la vivienda de la familia. Por ello, se retoman los planteamientos de Rodríguez-Triana (2018), quien resalta que la escuela debe posibilitar escenarios de construcción democrática de cultura, donde los individuos se formen desde la autorreferencia de los significados vivenciales, emocionales, prácticos y motivacionales, compartidos en la convivencia escolar y familiar; lo anterior, a través de un proceso de comunicación que facilite la protección de los procesos de enseñanza-aprendizaje (Metts \& Asbury, 2015). Por tanto, "el desafío de la escuela es atender la diversidad que tiene ante sí, desde la heterogeneidad y no de la homogeneidad como se hacía hasta hace unos años" (Martínez-Pérez, 2012, p. 52). En este sentido, es fundamental que las escuelas y familias coconstruyan un significado de participación unificado, a partir del diálogo, de tal manera que conceptos como implicación, participación y colaboración sean claros a la hora de establecer los canales comunicativos entre ambos sistemas (Martínez-Pérez, 2012); en consonancia con GoyoMorillo (2017), la escuela tiene el encargo de acoger a quien recién llega con su sistema de creencias.

De acuerdo con las experiencias previas, el tipo y nivel de implicación que las familias 
mantengan con el establecimiento educativo, se definirán algunos perfiles en esta relación (Martínez-Pérez, 2012). Para efectos del presente artículo, las clasificaciones de participación familiar encontradas permiten evidenciar posturas símiles y divergentes por parte de los autores, las cuales se agrupan en tres tendencias que se presentan a continuación (ver tabla 3).

\section{Tabla 3}

Tendencias en la clasificación de los niveles de participación familiar

\begin{tabular}{|c|c|c|}
\hline Tendencia & Indicador de la tendencia & Autores \\
\hline 1 & $\begin{array}{l}\text { 1. Informativa } \\
\text { 2. Consultiva: comunicación limitada, control } \\
\text { 3. Participación plena: verdadera comunicación escuela- } \\
\text { familia }\end{array}$ & Esteban (2015) \\
\hline 2 & $\begin{array}{ll}\text { 1. } & \text { Informativa } \\
\text { 2. } & \text { Consultiva } \\
\text { 3. } & \text { Decisoria } \\
\text { 4. Evaluativa } \\
\text { 5. Educativa }\end{array}$ & García-Carmona (2014) \\
\hline 3 & $\begin{array}{l}\text { 1. Parenting (Paternidad) } \\
\text { 2. Communicating (Comunicado) } \\
\text { 3.Volunteering (Trabajar como voluntario) } \\
\text { 4. Learning at Home (Aprender desde casa) } \\
\text { 5. Decision Making (Toma de decisiones) } \\
\text { 6.Collaborating with the Community } \\
\text { comunitaria) }\end{array}$ & $\begin{array}{l}\text { Wee } \\
(2011) \\
\text { Barnett } \\
(2016)\end{array}$ \\
\hline
\end{tabular}

Nota: elaboración propia.

De la tabla 3, se permiten agrupar y construir los tres conceptos fundantes para el desarrollo de este artículo, relacionados directamente con la participación de las familias; el primero de ellos es la implicación familiar, referida al grado de motivación y percepción y de afectación del proceso educativo de los hijos; la implicación depende entonces de las expectativas, intereses y necesidades de participación de cada contexto familiar (OchoaNinapaitán, 2018). Para Aguerri (2017), no es suficiente con la motivación y las percepciones familiares para hacer efectiva la participación, en tanto esta requiere de formación, la cual debe ser facilitada desde la estructura organizativa que poseen los canales de comunicación escuelafamilia, informales y formales. La implicación familiar podría incluirse aspectos como la 
paternidad, la tendencia informativa y consultiva.

El segundo concepto es el de participación familiar; el concepto de participación puede definirse como "una actitud y un interés solidario de apertura al diálogo, de preocupación por la vida colectiva en la que los individuos actúan motivados por la necesidad de mejorar sus condiciones de vida" (Márquez-Ibarra, 2014, p. 38), en cuanto hace referencia al "involucramiento de los padres, madres de familia en una o varias actividades relacionadas con la escuela" (Ochoa-Ninapaitán, 2018, p. 26). El nivel de participación familiar deberá entonces comprenderse como una actitud y un interés solidario por parte de los actores escolares en el mejoramiento de la calidad de vida de los niños y niñas y sus familias, a partir de la identificación de expectativas, intereses y necesidades de estos a intervenirse desde la institución escolar.

Finalmente, el tercer concepto: colaboración, recoge los niveles de participación evaluativa, decisoria, educativa y colaboración comunitaria. Este concepto integra aquellas acciones conducentes a crear territorio de encuentro y transformación institucional en la relación de familia y escuela, como lo pueden ser las escuelas de familia.

Sin embargo, es pertinente destacar que se pueden presentar algunas dificultades para establecer niveles óptimos de participación familiar, debido a los siguientes factores: a) la descontextualización de las acciones llevadas a cabo desde la escuela, en relación con las realidades de las familias; b) la delegación de responsabilidades al cuerpo profesorado, sin reconocimiento o remuneración extra; y c) la falta de claridades metodológicas para la planeación de acciones (Rodríguez-Triana y Suárez-Ortíz, 2019).

Al respecto de las dificultades o barreras que interfieren en la participación de las familias en la escuela, se pueden proponer algunas posibilidades de transformación y que pueden materializarse en actividades concretas, tal como propone Martínez-Pérez (2012) sobre el aprovechamiento de espacios y momentos compartidos, como las entregas de notas o boletines, para establecer puntos de encuentros en metas y estrategias metodológicas de familias y docentes. Por ende, para finalizar, la apertura de los escenarios educativos y del aula de clase como un territorio de construcción, que podría coadyuvar en el trabajo entre escuela y familia, sugiere que desde el ámbito organizativo de la escuela se deben adelantar estrategias de tipo grupal mediante el desarrollo de acciones participativas que involucren a los padres de familia, 
los docentes, y los directivos docentes, a través de la conformación, desarrollo y fortalecimiento de las escuelas de padres o escuelas de familia (Navarro-Perales, 1999).

\section{Escuelas de familia}

La familia tiene un rol protagónico en la socialización de las nuevas generaciones, pero no es posible abandonar otros escenarios de la vida cotidiana que desarrollan esta misma actividad; uno de ellos es la escuela, institución que desde los primeros años de vida se encarga de la formación de capital humano y social para la sociedad, y donde se inicia con la concreción de lo familiar en la sociedad. Es por ello importante en este punto preguntarse por las maneras de interrelación entre familia y la institución-escuela, para así comprender su horizonte formativo que va a integrar el desarrollo de capacidades, valores, conocimientos y habilidades sociales, necesarias en la formación intergeneracional.

Este reto que se plantea va a permitir pensar en las escuelas de familia como parte de la respuesta a este horizonte formativo, el cual va a establecer retos para los actores institucionales y para las familias. Con este concepto se hace referencia a los escenarios de reflexión y diálogo entre los diferentes actores educativos (docentes, familias y estudiantes), cuyo fin es el reconocimiento de las realidades individuales, familiares y colectivas que marcan riesgos, que inhiben, o posibilidades que potencian su desarrollo humano. Todo ello en el marco de la primacía de los derechos de los niñas y niñas, que se armonizan en el proyecto educativo institucional (PEI) de cada institución educativa.

La búsqueda de una relación armonizadora entre familia-escuela, permite reconocer la multiplicidad de formas familiares y sus contextos para el desarrollo democrático e incluyente de las capacidades necesarias para mejorar las condiciones del proceso de desarrollo de todos los

participantes, en especial de los niños y niñas (Ospina y Montoya, 2017). En otras palabras, "la escuela debe ser un espejo, es decir, un lugar en el que los chicos y chicas vean reflejada a su familia, y una ventana, puesto que además debe mostrar la diversidad familiar de nuestra sociedad" (Rodríguez-Mena, 2015, p. 88). Si bien, se apropia el término de escuelas de familia, sobre la base de ser una expresión que incluye el reconocimiento de la heterogeneidad de las familias, como principio de la acción educativa, es importante traer a la discusión el 
planteamiento de Cano-Muñoz (2015), quien va a comprender las escuelas de padres y madres de la siguiente manera:

Un grupo de aprendizaje pequeño, heterogéneo, libre, democrático e informal; un grupo que es capaz de participar de algún modo en la elección de los temas y colaborar en la organización de la propia escuela; un grupo que se autocritica y es capaz de tomar decisiones porque no se trata de teorizar, sino de llegar a potenciar las actitudes personales; un grupo que no prescinde en absoluto de los contenidos, pero que tiene un objetivo de cambio en las actitudes de cada persona consigo mismo y con su interacción con los demás. (p. 168)

Pensar la escuela de padres y madres como un espacio de aprendizaje colectivo para la formación de ser humano, más allá de los contenidos, permite en palabras de López-Soler (2010), la estructuración de una perspectiva global y coordinada de participación en estos espacios. Por tanto, esta debe contemplar factores históricos y sociales que den respuesta más eficaz a las intervenciones educativas.

De acuerdo con Cano-Muñoz (2015), una de las principales dificultades de la participación en las escuelas de familias radica en las múltiples miradas a lo que se realiza en estos encuentros. De acuerdo con Cano-Muñoz (2015) se encuentran tres tipos de participaciones, cada una con sus características y miradas frente al lugar de los padres de familia y los docentes de las instituciones educativas. En la primera, se encuentran cuidadores e instituciones que reducen las acciones de las escuelas de familia a términos operativos y didácticos, como talleres o actividades de entrenamiento; en la segunda, entienden el término como un conjunto de acciones desde la terapia familiar; y una tercera, atribuye las escuelas de familia a un programa de enseñanza unilateral y directiva que enseña a ser padres. Esta última idea puede crear ambientes de competición y desconfianza entre escuela-familia, y como resultado presentarse recelos por las contingencias en el proceso educativo de los estudiantes; además de un distanciamiento progresivo entre estas, en relación con los procesos de socialización que cada una desarrolla y que en el ambiente escolar se integran (Gallego, 2016).

Al respecto, Jiménez-Martínez (2015) ubica dos perspectivas para entender las dificultades a las que se enfrentan las familias para participar en estos espacios de construcción; la primera se relaciona con obstáculos propios de cada familia, con respecto a su enrolamiento 
con el medio educativo. La segunda comprende las dificultades en la participación de las familias en los establecimientos educativos; para este caso y retomando a Jiménez-Martínez (2015), se encuentran una serie de factores relacionados con los niveles socioeconómicos, ciclo evolutivo de los padres y madres de familia, disponibilidad y claridad en la información relacionada con la escuela y la importancia de su participación, dificultad de conciliar el tiempo de trabajo con el familiar y de participación institucional.

Finalmente, de acuerdo con Carmona (2014), Capera y Torres (2014) y Jiménez Martínez (2015) la participación de las familias en los entornos escolares, a través de las escuelas familiares puede clasificarse de la siguiente manera:

Familias con problemas: caracterizadas, en primer lugar, por las dificultades socioeconómicas por las cuales se segregan de los procesos participativos de la escuela; las limitaciones socioeconómicas que parten de las clases sociales crean tres barreras para la participación de las familias en la escuela: en primer lugar, responde a que la escala de prioridades de las familias en situación de pobreza no incluye el acompañamiento y el desarrollo educativo formal de los hijos (Carmona, 2014). Al respecto, Capera y Torres (2014) mencionan que el analfabetismo se puede traducir en menores expectativas educativas de los padres y madres con sus propias vidas y las de los hijos; no obstante, los mismos autores hacen la salvedad que algunas familias tienen una clara intención de apoyar el proceso formativo de sus hijos, pero carecen de las herramientas necesarias para hacerlo. En segundo lugar, las familias con mayores niveles educativos pueden establecer relaciones más simétricas con el entorno escolar; y, en tercer lugar, la escuela limita la participación de las familias más vulnerables, en cuanto las ubica como deficientes (Carmona, 2014). Gallego (2016) recoge diferentes aportaciones de autores y estudios en los cuales el nivel socioeconómico no es determinante para el éxito escolar, si bien puede ser una variable importante, pareciera que las conductas educativas desarrolladas desde las familias son predictores más potentes para el acompañamiento de niños y niñas en la escolaridad.

Familias centradas en los niños: estas tienden a sobre-implicarse en las dinámicas de las instituciones educativas, con el fin de proteger a sus hijos; pueden llegar a culpabilizar o desvalorizar la labor de otros adultos y educadores; si bien la asignación de roles relacionados a 
la actividad escolar puede ser beneficioso para quienes las integran, sus aportaciones pueden percibirse como una amenaza en el ambiente institucional (Jiménez-Martínez, 2015).

Familias centradas en los padres: se caracterizan por tener padres con altos recursos educativos y culturales; la participación en los procesos educativos formales de sus hijos se ve relegada, de cara a sus proyectos profesionales y personales. Estos padres y madres pueden resultar de gran ayuda, siempre y cuando se les inste a participar en roles que les posibilite explotar sus habilidades y conocimientos (Jiménez-Martínez, 2015).

Familias ausentes o desinteresadas: se trata de cuidadores desligados del establecimiento educativo, con encuentros mínimos con los docentes; en su lugar acuden familiares cercanos que no tienen poder decisivo frente a los asuntos escolares y, generalmente, se justifican por la falta de tiempo (Jiménez-Martínez, 2015).

Familias participativas: familias con expectativas altas en el desarrollo educativo de los hijos, y con implicación alta en la escuela (Jiménez-Martínez, 2015).

\section{Educación afectivo-sexual: entre lo público y lo privado}

Para comprender la educación afectivo-sexual es necesario escudriñar en el concepto de sexualidad y afectividad. En primer lugar, se puede decir que la sexualidad humana es el resultado de la interacción cognitiva y afectiva de los individuos y su medio o cultura, respecto a las posibilidades de expresión de sus comportamientos sexuales, afectivos, placenteros y filogenéticos (Barragán-Medero, 1991); dicha interacción, según Rogoff (1997), fomenta vínculos de dependencia y afecto en un entorno común.

Es así como en Colombia, la Política Nacional de Salud Sexual y Reproductiva (Ministerio de Protección Social, 2003) plantea, en el componente de Salud Sexual y Reproductivas, que "la sexualidad y la reproducción y la forma en que éstas se viven están determinadas por condicionamientos culturales y sociales que es posible deconstruir en el marco del proyecto de vida que cada quien elige para sî".

En segundo lugar, la afectividad es un concepto referido al conjunto de características de comunicación, placer, roles de género, valores, relaciones interpersonales, entre otros aspectos relacionales (López-Soler, 2010). Así, la afectividad requiere de tres condiciones: (1) seguridad 
emocional, (2) intimidad y contacto corporal, y (3) apoyo social (López-Soler, 2010). Estas condiciones se materializan en la relación con la familia, la escuela y su entorno.

Ahora bien, cuando se habla del concepto de educación afectivo-sexual se ha de tener en cuenta dos componentes de la educación, tal y como se mencionó en el apartado de escuela: territorio de encuentro; esta puede ser instructiva y formativa, y se refiere a la trasmisión de información de los aspectos biológicos y fisiológicos de la sexualidad. En cuanto a la educación formativa, abarca aspectos más profundos de la comunicación humana, como la transmisión de actitudes y valores (López-Soler, 2010). Ambos componentes de la educación serían fundamentales en el desarrollo de estrategias educativas en afectividad y sexualidad. Sin embargo, es necesario considerar algunos de los factores que pueden incidir en el proceso y que requieren ser atendidos ante cualquier intento para formular acciones concretas:

Factores culturales y territoriales: los aspectos relacionados con la ubicación espacial de las familias, estudiantes y docentes; el nivel socioeconómico y los valores culturales definen en qué temas se educa, cuándo y cómo se abordan los diálogos respecto a la sexualidad; de igual forma, otros determinantes socioculturales, como los medios de comunicación, la relación con sus pares, la religión, y otros aspectos constitutivos de la vida escolar, ocupan un lugar importante en este proceso de construcción de significados en el cuerpo y la vida humana (López-Beltrán, 2016). En este sentido, las nuevas formas de intimidad y extimidad presentan limites borrosos en un mundo donde las maneras de relacionarse son determinadas en buena medida por el uso de tecnologías de la información determinan en buena medida las maneras de relacionarse. Lo anterior, va a permitir interrogar lo pedagógico desde la perspectiva de la educación afectivo-sexual (Valdivielso et al., 2016).

Factores relacionados con el género: el género como unidad de análisis ha de contemplarse desde el ámbito familiar y escolar; para el primero, López-Beltrán (2016) apunta a que los lugares desde los que se educa están influenciados por los roles de género imperantes. Para el caso de las madres, o quien ocupa su función, hay una mayor tendencia a la educación desde el diálogo; en contraparte, el padre, o quien ocupe el lugar y función de paternar, parece estar más alejado de la pedagogía relacionada con la educación afectiva y sexual; igualmente, los contenidos de la educación están influenciados por los estereotipos de género, en tanto se puede evidenciar, por ejemplo, que en las culturas conservadoras la educación y responsabilización de 
la reproducción y prevención de las infecciones de transmisión sexual están dirigidas a las hijas (López-Beltrán, 2016).

Para el caso de la institucionalidad escolar, el currículum puede jugar el papel de instrumento, en tanto que no las considera en los diseños metodológicos y pedagógicos, y por el contrario se escribe y ejecuta en términos de hegemonías sexuales, de género, socioeconómicas, y posibilidades físicas y cognitivas (Valdivielso et al., 2016).

Factores relacionados con el perfil de los estudiantes-hijos/as: de acuerdo con LópezBeltrán (2016), los contenidos y metodologías, adoptados por familias e instituciones en la educación afectivo-sexual, deben adaptarse a las condiciones particulares de los educandos, hijos e hijas; con esto, se refiere al ciclo evolutivo en el que se encuentran, contexto social, conocimientos y valores previos, o cualquier otra situación diferenciadora; lo anterior, con la finalidad de que los estudiantes, hijos e hijas, obtengan una educación de calidad de acuerdo con sus necesidades (López-Beltrán, 2016).

A partir de lo anterior, es importante preguntarse por las acciones conjuntas que permitan dar un horizonte integrativo de familia y escuela en la educación sexual y afectiva. Las acciones compartidas a través de estas se convierten en un ejercicio de transformación cuando el vínculo escuela-familia es sostenido por la confianza y la corresponsabilidad entre quienes hacen parte de la comunidad educativa; al partir del reconocimiento de la familia como agente social capaz de generar virtudes imprescindibles de la humanidad, como la confianza, que posteriormente como capital social, pueden ser desarrolladas en el ámbito público de la institucionalidad, como lo es la escuela (Ros-Codoñer, 2018).

No se puede desconocer que estas funciones generadoras de capital social están indefectiblemente ligadas a los entornos educativos, tal y como lo menciona Rodríguez-Triana (2018) al sugerir que la institucionalidad tiene el compromiso de reconocer y articular las experiencias familiares y aprendizajes de los estudiantes al aula, desde la indagación y recuperación de las prácticas educativas de la familia, entendidas como acciones significativas de la cotidianidad, que dirigen el comportamiento social y se gestan desde el encuentro de subjetividades de los miembros de la unidad familiar en una trama relacional, plural y diversa.

Por lo anterior, la escuela, además de la transmisión de aprendizajes formales, debe emprender un camino hacia la trascendencia, en aras de constituirse en un territorio para el 
encuentro; así lo mencionan Rogoff et al. (2001), al referirse a las prácticas educativas de orientación dialógica para aumentar el aprendizaje y mejorar la convivencia, y donde las familias se identifiquen con posibilidades diversas que permitan potenciar sus capacidades y superar las barreras que dificultan la acción articulada en educación afectivo-sexual con el establecimiento educativo. En relación con este punto, González-Gómez (2015) menciona que:

El reto está en reconocer las sexualidades como un valor de los seres humanos. La sexualidad no debe verse como un escenario de disputa y exclusión, sino como un espacio de aprendizaje y creación, para ello, necesariamente, debe atravesar nuestra intimidad y nuestro cuerpo nos debe posibilitar un re-conocimiento de nuestros miedos, ausencias, resistencias, goces, deseos y desconocimientos. (p. 13)

De acuerdo con los anteriores planteamientos, las escuelas de familia se proponen, desde este artículo, como una posibilidad de conciliar los espacios públicos y privados de estudiantes, hijos e hijas, siendo estos el centro de la interrelación familia-escuela. En palabras de Esteban (2015), se hace necesario analizar los beneficios de la acción articulada de familia y escuela para los y las estudiantes, desde tres frentes: el primero, relacionado con el rendimiento académico; en segundo lugar, la motivación y la actitud que logren, de cara a la escolaridad; y, por último, considerar los beneficios afectivos y psicológicos. Para efecto de este artículo se hará referencia a los dos últimos.

En cuanto a la dimensión afectiva y psicológica de los estudiantes, esta se ve positivamente influenciada por la integración de las acciones educativas familia-escuela; favorece el desarrollo de la personalidad en niños y niñas, la autoestima, confianza y seguridad social (Esteban, 2015).

En relación con los beneficios en la diada familia-escuela, se puede decir que en este proceso logran desarrollar actitudes y habilidades necesarias para ejercer la responsabilidad de la crianza, en complementariedad de la educación formal, ya que esta contribuye a la contextualización y materialización de los aprendizajes fomentados desde las prácticas familiares, al tiempo que el aprendizaje, visto como proceso regulatorio, no es considerado una tarea sencilla (Villach y Llanos, 2017). De igual manera, la familia tiene la posibilidad de garantizar que las necesidades de sus hijos e hijas sean atendidas desde la escolaridad, puesto que la escuela como entorno socializador primario (Berger y Luckmann, 1999), tiene la información 
más relevante sobre el alumno (Esteban, 2015). A su vez, Castro-Zubizarreta y García-Ruíz (2016) mencionan como "otros beneficios que se encuentran, aunque en menor medida, son el incremento de sensaciones de seguridad y la percepción de una mayor competencia, habilidad en la educación de los hijos" (p. 204). Dicha percepción de mayor competencia se incrementa en la medida que la comunicación de padres, madres, hijos e hijas mejora como parte del compromiso compartido en la educación (Martínez-Pérez, 2012).

La concurrencia entre familia y escuela permite que las acciones desarrolladas en el ámbito formal, dirigidas a la educación afectivo-sexual, se contextualicen con las necesidades de estudiantes y familias, lo cual genera resultados óptimos y mejora la percepción del profesorado sobre su propia gestión en el contexto educativo (Esteban, 2015). Una puntada final, y a modo de finalización, en las voces de autores como Burke \& Hodapp (2014), Heiman et al. (1998), Kippax et al. (2005) y Kouta \& Tolma (2008), quienes esbozan el lugar identitario de la sexualidad en los niños, niñas y adolescentes en la familia y el territorio escuela; en ambos lugares aparece la educación y la formación en asíntotas vitales en la acción integral e integradora del ser humano en el ambiente educativo.

\section{Discusión}

El desarrollo argumentativo, construido frente a la relación escuela-familia y educación afectivo-sexual, permite identificar la relevancia de las escuelas familiares como escenarios para la construcción de procesos, en los cuales se permite la construcción de una conciencia reflexiva frente los derechos sexuales y reproductivos de hombres y mujeres en los espacios educativos. Se requiere, entonces, el compromiso de docentes y padres de familia para continuar fortaleciendo el capital humano en formación. Es por ello importante recordar el planteamiento del Fondo de Población de Naciones Unidas (2016), con respecto a este asunto:

Las decisiones sexuales y reproductivas de los y las jóvenes pueden tener un efecto cascada sobre sus derechos humanos. El embarazo adolescente, por ejemplo, puede llevar a las jóvenes a abandonar la escuela, privándolas así de su derecho a la educación. (p. 9)

En este orden de ideas, la familia, la escuela, los docentes y la sociedad son corresponsables en la educación de las nuevas generaciones y están llamados a la acción, a través 
del diálogo y la construcción de significados, para responder cuestiones como: ¿en qué educar?, ¿cuándo educar?, ¿cómo educar?, ¿para qué educar? Interrogantes de orden ontológico frente al proceso de enseñanza-aprendizaje que debe ser siempre foco de atención por parte de los padres de familia, y de discusión, revisión y análisis en los espacios denominados escuelas familiares.

A partir de esta línea reflexiva de carácter argumentativo, se hace necesario articular a las escuelas familiares una apuesta metodológica y práctica, en la cual se desarrolle una concreción de acciones relacionadas con la educación afectivo-sexual. Para este caso se hará referencia al enfoque basado en los derechos humanos; apuesta que permitirá en su desarrollo el análisis y corrección de las desigualdades en los contextos de acción donde se desarrollen (Langa-Herrero, 2019). Ello implica reconocer una mirada amplia de estos, que involucran lo individual y lo colectivo, pero también una mira holística a los derechos, pero haciendo énfasis en los sexuales y reproductivos, esenciales para la comprensión de la educación sexual-afectiva en la escuela; "pudiéramos decir que los derechos sexuales constituyen la personalización más accesible para que los individuos se apropien de los derechos humanos" (Collignon-Goribar y Lazo-Corvera, 2017, p. 59).

Esta articulación del enfoque de derecho está articulada a cuatro enfoques afines: enfoque de género, diferencial, territorial y la acción sin daño, los cuales es necesario profundizar para seguir ampliando el radio de acción de las escuelas familiares, en cuanto a la educación afectivosexual. El enfoque de género, como una unidad de análisis, permite vislumbrar las disparidades de poder entre hombres y mujeres, lo que corresponde a un trasfondo estructural (BarragánGamba, 2015); este enfoque ha permitido trascender en asuntos más profundos que la disparidad de polaridades, y ha dispuesto el camino hacia nuevas discusiones sobre formas de ser humano, más allá de la feminidad y masculinidad hegemónica, enmarcada en la heteronormatividad (Talani-Zuvela, 2019); esta categoría analítica permite que los adultos involucrados en el proceso de la educación afectivo-sexual, "sean autocríticos con sus propios sesgos en la enseñanza, como también que puedan ser agentes de transformación de los roles tradicionales de género." (Barrientos et al., 2018, p. 10).

El enfoque diferencial es parte fundamental del ejercicio pedagógico, dado que parte de las diferencias como garantía de las acciones equitativas, y se consideran condiciones como situación o condición de discapacidad, edad, identidad de género, diversidad sexual, nivel 
socioeconómico, diversidad étnica, entre otros aspectos relevantes en la configuración de las subjetividades participantes (Secretaría de las Mujeres de Antioquia, 2020). El enfoque territorial, también se estima dentro de las acciones de la educación afectivo-sexual desde las escuelas de familia, en tanto permite analizar las condiciones del contexto real en donde las escuelas de familias van a tener lugar; más allá de los espacios físicos, el territorio cuenta con una representación simbólica en lo psicosocial, por lo que las acciones que desde este enfoque se adelanten tienen "como objetivo solucionar problemas comunes de acuerdo a los intereses y prioridades de un territorio socialmente construido, con identidad propia de amplia interrelación y consenso entre agentes" (Garín-Diez et al., 2016, p. 29).

Por último, el enfoque de acción sin daño (Do no Harm) es una consideración ética y sensible a los conflictos y posibles escenarios o contextos de violencia, donde la población participante puede ubicarse; en este caso las acciones concebidas desde la planificación y acción deben contemplar posibles efectos positivos o negativos para la atención de dicho conflicto, cuyo fin último es salvaguardar a las instituciones educativas, docentes, familias y estudiantes de un mal mayor que pudiese ocasionarse (Langa-Herrero, 2019).

En virtud de lo señalado, el enfoque basado en los derechos humanos permitiría abordar los temas y características del trabajo a desarrollar desde las escuelas de familia en educación afectivo-sexual, como proceso basado en el aprendizaje cooperativo y continuo entre escuelafamilia. De tal manera, en palabras de Freire (2002) pensar en la esperanza del trabajo articulado familia-escuela y la garantía de la educación afectivo-sexual integral para niños y niñas se vuelva historia.

\section{Conclusión}

La revisión teórica desarrollada en este escrito posibilitó comprender las escuelas de familia como escenario de encuentro entre los espacios públicos y privados, en los cuales se generan procesos interactivos y de intercambio entre los diferentes actores escolares, en especial entre la diada familia-escuela. Es por ello importante resaltar la necesidad de desarrollar apuestas teóricas y metodológicas que permitan ampliar estas lecturas contextuales, que para este caso fue el enfoque de derechos, eje transversal que favorece la construcción de significados y diálogo entre la familia, 
la escuela, los docentes y los padres de familia, sin dejar de lado el enfoque de género, diferencial, territorial y acción sin daño, en la medida en que la planeación de acciones, desde estas perspectivas integradas, favorecerán un mayor acceso para que los individuos se apropien de los derechos humanos.

Por ello, es importante señalar que el abordar la educación afectivo-sexual desde el enfoque de derechos, debe permitir espacios en los cuales las escuelas de familia tengan un lugar privilegiado en su desarrollo, en cuanto a que hoy se enfrenta el reto de encarar la diversidad en tipologías de familia, contextos, representaciones y estereotipos del género, que se interseccionan e interconectan. Por tanto, este estado del arte permite recrear una propuesta educativa, en clave de los derechos sexuales y reproductivos, la cual consiste en vislumbrar la diferencia que todos y todas ocupan, y desde la cual se invita a abordar las decisiones y la participación de docentes, familias y estudiantes como una posición política de la experiencia, puesta en coherencia con la construcción de espacios más sensibles a la inclusión y al cambio.

Hacer referencia a la inclusión y al cambio, como apuesta de las escuelas familiares, es posible desde la apropiación que se realizó de las cuatro categorías desarrolladas en el escrito: lo familiar en la sociedad, en la cual se analizó la naturaleza multidimensional y multicausal de las comprensiones de familia, y la directa relación que existe entre estas y las actuaciones profesionales. Subrayando de esta manera, la importancia de reconocer las historias, prácticas y discursos, así como las capacidades y emociones que constituyen los sujetos que integran las familias. De otro lado, la categoría escuela: territorio para el encuentro, que facilitó encontrar la interrelación entre la escuela y la familia, en términos de posibilitar escenarios de construcción democrática de cultura, y atender la diversidad y heterogeneidad de los niños, niñas y adolescentes y sus familias, a la luz de conceptos como implicación familiar, participación familiar y colaboración; cada uno de estos resultado de la construcción teórica-conceptual de los documentos revisados en el estado del arte.

Con respecto a las escuelas de familia, tercera categoría resultado del estado del arte, se subraya la participación de las familias como un espacio para la construcción de experiencias formativas, en las cuales se deben tener en cuenta factores relacionados con el nivel socioeconómico, el curso de vida de los padres, los escolares y las condiciones de relación y vinculación con esta propuesta de convergencia de la familia-escuela. En cuarto lugar, la educación 
afectivo-sexual: entre lo público y lo privado, a través de la cual se logró una interrelación entre los componentes de la educación y la formulación de acciones relacionadas con: a) factores culturales y territoriales, b) factores relacionados con el género, y c) factores relacionados con el perfil de los estudiantes-hijos/as. Esto mostró un horizonte integrativo entre escuela-familia como territorio para el encuentro entre la educación sexual y afectiva.

Finalmente, este estado del arte deja abierta la discusión sobre la triada familia, educación afectivo-sexual y escuela, ya que resulta ser un reto para realizar y potenciar, en términos de la interacción social y comunicativa (García, 2016). Para cerrar, es importante resaltar la interactividad, la construcción de conocimiento en las aulas y escuelas familiares como engranajes que tendrán influencia en el desarrollo educativo de los niños y niñas (Coll et al., 1992).

\section{Referencias}

Aguerri, L. M. R. (2017). Comunidades de aprendizaje y participación familiar: un estudio de casos (Tesis de doctorado). Universidad Pablo de Olavide. https://rio.upo.es/xmlui/handle/10433/4806

Barnett, T. M. (2016). Access, technology, and parental involvement: A case study on a west Los Angeles charter school (Doctoral dissertation). Loyola Marymount University. https://digitalcommons.Imu.edu/cgi/viewcontent.cgi?article=1467\&context=etd

Barragán-Gamba, D. (2015). La violencia contra las mujeres es cuestión de género. Criterio Libre Jurídico, 12(2), 79-90. https://doi.org/10.18041/crilibjur.2015.v12n2.24203

Barragán-Medero, F. (1991). Programa de Educación Afectivo Sexual. Educación Secundaria I.

Sexualidad, educación sexual y género. Junta de Andalucía, Consejería de Educación y Ciencia e Instituto Andaluz de la Mujer.

Barrera, D., y Ortiz, M. (febrero-mayo, 2017). La Red de Escuelas de Música de Medellín: un espacio para la construcción de la identidad de los adolescentes participantes. Revista Virtual Universidad Católica del Norte, (50), 238-255. http://revistavirtual.ucn.edu.co/index.php/RevistaUCN/article/view/822/1340

Barrientos, P., Andrade, D., y Montenegro, C. (2018). La formación docente en género y diversidad sexual: Tareas pendientes. Cuaderno de Educación, (81), 1-13. 
http://cuadernosdeeducacion.uahurtado.cl/81/

Berger, P. L., y Luckmann T. (1999). La construcción social de la realidad. Amorrortu editores.

Burke, M. M., \& Hodapp, R. M. (2014). Relating Stress of Mothers of Children With Developmental Disabilities to Family-School Partnerships. Intellectual \& Developmental Disabilities, 52(1), 13-23. https://doi-org.consultaremota.upb.edu.co:8443/10.1352/19349556-52.1.13

Cano-Muñoz, M. (2015). Sentido y fundamento de las Escuelas de Padres y Madres: orientaciones para una responsabilidad compartida (Tesis de doctorado). Universidad Complutense de Madrid. https://eprints.ucm.es/id/eprint/30797/1/T36192.pdf

Capera, I., y Torres, A. (2014). Sentidos y prácticas frente a la relación Familia-escuela en comunidades indígenas etnias: "Nasa" de Cajibío-Cauca y "Pijao" de Coyaima-Tolima. (Tesis de maestría). Universidad de Manizales.

Carmona, C. A. (2014). Familia, escuela y clase social: sobre los efectos perversos de la implicación familiar. Revista de Sociología de la Educación-RASE, 7(2), 395-409. https://dialnet.unirioja.es/servlet/articulo?codigo=5144522

Carvajal, B. M., Castro, O. C., y Aguilera, R. B. (2018). Análisis histórico de la orientación a la familia en niños de la infancia preescolar, en Cuba. Conrado, 14(62), 32-38. https://conrado.ucf.edu.cu/index.php/conrado/article/view/675/709

Castro-Zubizarreta, A., y García-Ruíz, R. (2016). Vínculos entre familia y escuela: visión de los maestros en formación. Magis. Revista Internacional de Investigación en Educación, 9(18), 193-208. https://www.redalyc.org/pdf/2810/281049122011.pdf

Coll, C., Colomina, R., Onrubia, J., y Rochera, M., J. (1992). Actividad conjunta y habla: una aproximación al estudio de los mecanismos de influencia educativa. Journal for the Study of Education and Development, Infancia y aprendizaje, 15(59-60), 189-232. https://doi.org/10.1080/02103702.1992.10822356

Collignon-Goribar, M. M., y Lazo-Corvera, P. (2017). Derechos humanos/sexuales, género y biopolítica: reflexiones sobre la configuración subjetiva del derecho a la libre elección de identidad de género. La ventana. Revista de estudios de género, 5(45), 50-87. http://www.scielo.org.mx/scielo.php?script=sci_arttext\&pid=S1405-

\section{0\&lng=es\&tlng=es}


Congreso de la República de Colombia. (16 de julio de 1996). Ley 294, por la cual se desarrolla el artículo 42 de la Constitución Política y se dictan normas para prevenir, remediar y sancionar la violencia intrafamiliar. https://www.icbf.gov.co/cargues/avance/docs/ley_0294_1996.htm

Congreso de la República de Colombia. (8 de noviembre 2006). Ley 1098, por la cual se expide el Código de la Infancia y la Adolescencia. https://www.icbf.gov.co/cargues/avance/docs/ley_1098_2006.htm

Congreso de la República de Colombia. (27 de julio de 2010). Ley 1404, por la cual se crea el programa escuela para padres y madres en las instituciones de educación preescolar, básica y media del país. http://www.secretariasenado.gov.co/senado/basedoc/ley_1404_2010.html Congreso de la República de Colombia. (23 de julio de 2020). Ley 2025, por medio de la cual se establecen lineamientos para la implementación de las escuelas para padres y madres de familia y cuidadores, en las instituciones de educación preescolar, básica y media del país, se deroga la ley 1404 de 2010 y se dictan otras disposiciones. https://dapre.presidencia.gov.co/normativa/normativa/LEY\%202025\%20DEL\%2023\%2 0DE\%20JULIO\%20DE\%202020.pdf

Dabas, E., Celma, L.C., Rivarola, T., y Richard, G.M. (2011). Haciendo en redes. Perspectivas desde prácticas saludables. Ediciones CICCUS.

Damiani, A. M. (2020). Sobre una contribución de Julio De Zan a la metodología de la hermenéutica filosófica. Erasmus. Revista para el Diálogo Intercultural, 22, 25-43. https://www.icala.org.ar/erasmus/Archivo/2020/erasmus\%2022-1-2020/erasmus-22-12020-05-Damiani.pdf

Denzin, N., y Lincoln, Y. (2012). Manual de investigación cualitativa vol. 1. El campo de la investigación cualitativa. Gedisa.

Durán de León, C. (2018). Programa de formación social para optimizar la integración familiaescuela-comunidad en educación primaria (Tesis de maestría). Universidad de Carabobo.

Esteban, A. (2015). Relación familia-escuela: Estudio descriptivo derivado de una experiencia de investigación-acción en el aula (Tesis doctoral). Universidad de Valladolid.

Fondo de Población de las Naciones Unidas. (2016). Educación sexual integral. https://www.unfpa.org/es/educaci\%C3\%B3n-sexual-integral 
Freire, P. (2002). Pedagogía de la esperanza. Un reencuentro con la pedagogía del oprimido. Siglo XXI Editores México.

Fúnez, D. (2014). La gestión escolar y la participación de los padres de familia en el proceso educativo de sus hijos (Tesis de maestría). Universidad Pedagógica Nacional Francisco Morazán.

Galián, N., García, M., P., y Belmonte, M., L. (2018). Evaluación de la participación familiar en el proceso educativo de los discentes desde la percepción del profesorado. Revista Electrónica Interuniversitaria de Formación del Profesorado, 21(3), 45-62. https://doi.org/10.6018/reifop.21.3.336971

Gallego, J. M. (2016). La familia y el desarrollo educativo de los hijos: una mirada sistémica. (Tesos de doctorado). Universidad de Alcalá.

García, M. (2016). La comunicación como base para la interacción social. Aportaciones de la comunicología al estudio de la ciudad, la identidad y la inmigración. Contemporânea, 2(2), 53-71. https://doi.org/10.12957/contemporanea.2004.23158

García-Carmona (2014). Análisis de las percepciones sobre liderazgo y participación de las familias en asociaciones de madres y padres en contextos multiculturales. Un estudio comparativo entre Nueva York y Granada (Tesis de doctorado). Universidad de Granada. http://hdl.handle.net/10481/51135

Garín-Diez, F., Meza-Lueyza, Y., y Plaza-Dote, A. (2016). Formación en derechos sexuales y reproductivos con enfoque de género (Tesis de pregrado). Universidad Academia de Humanismo Cristiano.

González-Gómez, Y. (2015). El papel del docente en la educación para la sexualidad: algunas reflexiones en el proceso educativo escolar. Actualidades Investigativas en Educación, 15(3), 1-15. https://revistas.ucr.ac.cr/index.php/aie/article/view/20335

Goyo-Morillo, W. (2017). Corresponsabilidad familia-escuela. Una Hermeneusis desde sus actores. Revista Multidisciplinaria DIALÓGICA, 14(1), 25-49. http://revistas.upel.edu.ve/index.php/dialogica/article/view/5768/3073

Guerrero-Bejarano, M. (2016). La Investigación Cualitativa. INNOVA Research Journal, 1(2), 1-9. https://repositorio.uide.edu.ec/bitstream/37000/3645/3/document.pdf

Guevara-Patiño, R. (2016). El estado del arte en la investigación: ¿análisis de los conocimientos 
acumulados o indagación por nuevos sentidos? Revista Folios, 44, 165-179. http://www.scielo.org.co/pdf/folios/n44/n44a11.pdf

Heiman, M. L., Leiblum, S., Esquilin, S. C., \& Pallitto, L. M. (1998). A Comparative Survey of Beliefs about "Normal" Childhood Sexual Behaviors. Child Abuse \& Neglect, 22(4), 289-304. https://doi-org.consultaremota.upb.edu.co:8443/10.1016/S01452134(97)00176-2

Horna-Clavo, E., Arhuis-Inca, W., y Bazalar-Palacios, J. (septiembre-diciembre, 2020). Relación de habilidades sociales y tipos de familia en preescolares: estudio de caso. Revista Virtual Universidad Católica del Norte, (61), 224-232. https://www.doi.org/10.35575/rvucn.n61a13

Issó-García, D. (2012). La participación de las familias en la escuela pública española (Tesis de doctorado). Universidad de Granada.

Jiménez-Martínez, M. (2015). La participación de la familia y otros agentes de la comunidad en la implementación de programas educativos en centros de educación primaria (Tesis de doctorado). Universidad de Murcia. http://hdl.handle.net/10803/336091

Kippax, S. Smith, G., Weaver, H. (2005). School Based Sex Education Policies and Indicators of Sexual Health Among Young People: A Comparison of the Netherlands, France, Australia, and the United States. Sex Education, 5(2), 171-188. https://doi.org/10.1080/14681810500038889

Kouta, C., \& Tolma, E. L. (2008). Sexuality, sexual and reproductive health: an exploration of the knowledge, attitudes and beliefs of the Greek-Cypriot adolescents. Promotion \& Education, 15(4), org.consultaremota.upb.edu.co:8443/10.1177/1025382308097695

Lacasa, P. (1997). Familias y escuelas. Caminos de la orientación educativa. Visor

Langa-Herrero, A. (2019). La incorporación de los derechos humanos a las metodologías de cooperación al desarrollo a través del enfoque de derechos y la acción sin daño. Revista Internacional de Pensamiento Político, 251-267. https://upo.es/revistas/index.php/ripp/article/view/4783

López-Beltrán, M., I. (2016). Actitudes y creencias de padres y madres hacia la educación sexual (Tesis de maestría). Universidad Jaume I. 
López-Soler., N. (2010). Curso de Educación Afectivo-Sexual: Libro de Teoría. Netbiblo, S.L.

Márquez-Ibarra, L. (2014) La participación social en la escuela secundaria pública. Los padres y madres de familia como actores sociales: un estudio de caso (Tesis de doctorado). UIA Puebla.

Martínez-Pérez, S. (2012). La relación escuela-familia, la representación de un espacio compartido (Tesis de doctorado). Universitat de Barcelona.

Metts, S., \& Asbury, B. (2015). Theoretical Approaches to Family Communication. En H. Lynn \& R. Turner. (Eds.). The SAGE Handbook of Family Communication. SAGE Publications.

Ministerio de la Protección Social. (2003). Política Nacional de Salud Sexual y Reproductiva. https://www.minsalud.gov.co/sites/rid/Paginas/freesearchresults.aspx ?k=\&k=salud\%20se $\underline{\text { xual }}$

Montoya-Ahmedt, C. A. (2017). La paternidad tras la ruptura de pareja: transformaciones derivadas de los procesos de separación. Revista Latinoamericana de Estudios de Familia, 9, 131-147. http://revlatinofamilia.ucaldas.edu.co/downloads/Rlef9 9.pdf

Navarro-Perales, M. (1999). Análisis de distintas estrategias para la participación de los padres en la escuela. Profesorado. Revista de Currículum y Formación del Profesorado, 3(1), 116. https://digibug.ugr.es/handle/10481/23139

Ochoa-Ninapaitán, H. (2018). Participación de los padres de familia como corresponsables de la educación de sus hijos en una institución educativa del Callao (Tesis de maestría). Universidad San Ignacio de Loyola.

Ospina, B., M., y Montoya, P., E. (2017). Las prácticas educativas con familia desde la escuela. Zona Próxima, (26), $31-43$. http://search.ebscohost.com.consultaremota.upb.edu.co/login.aspx?direct=true\&db=ehh \&AN=124715840\&lang=es\&site=ehost-live

Puyana, Y. (2007). El familismo: una crítica desde la perspectiva de género y el feminismo. En Puyana, Y. y Ramírez, M. H. (Eds.), Familias, cambios y estrategias (422). Universidad Nacional de Colombia, Alcaldía Mayor de Bogotá y Secretaría Distrital de Integración Social.

Rivera Sepúlveda, Á. A., y Guerra García, Y. M. (septiembre-diciembre, 2020). Tensiones y 
discontinuidades en la comprensión comunitaria de la escuela rural. Revista Virtual Universidad Católica del Norte, (61), 102-121. https://www.doi.org/10.35575/rvucn.n61a7

Rodríguez-Mena, J. (2015). Familias homoparentales en la escuela pública: Estudio de caso de la intervención escolar sobre la diversidad familiar (Tesis de doctorado). Universidad de Huelva.

Rodríguez-Triana, Z. E. (2018). Qué y cómo se enseña y aprende en la familia. Un asunto de interés para la escuela. Revista Latinoamericana de Estudios Educativos, 14(2), 132-157. http://latinoamericana.ucaldas.edu.co/downloads/Latinoamericana14(2)_7.pdf

Rodríguez-Triana, Z., y Suárez-Ortíz, J. (2019). Escuelas de Familia. Una experiencia favorecedora para el desarrollo humano. Revista Electrónica Interuniversitaria de $\begin{array}{llll}\text { Formación } & \text { del } & \text { Profesorado, } & \text { 22(3), }\end{array}$ https://revistas.um.es/reifop/article/view/390161

Rogoff, B. (1997). Los tres planos de la actividad sociocultural: apropiación participativa, participación guiada y aprendizaje. En J. Wertsch, P. del Río y A. Álvarez (Eds.), La mente sociocultural. Aproximaciones teóricas y aplicadas (pp. 111-128). Fundación Infancia y Aprendizaje.

Rogoff, B., Turkanis, G., \& Bartlett, L. (2001). Learning together: Children and adults in a school community. Oxford University Press.

Ros-Codoñer, J. (2018). La familia, clave en la construcción de la sociedad de lo humano. En C. Arango y E. Calderón (Coords.), Desarrollo y territorio III: Comunidad, familia y educación (pp. 219-230). Fondo editorial Universidad Católica de Oriente.

Secretaría de las Mujeres de Antioquia. (2020). Actualización: Plan para Erradicar la Violencia Contra las Mujeres. Gobernación de Antioquia. https://antioquia.gov.co/index.php/secretaria-de-las-mujeres-de-antioquia

Talani-Zuvela, P. (2019). Educación sexual integral y perspectiva de género en la formación docente: una conjunción indispensable para una pedagogía crítica y feminista. Saberes y prácticas. Revista de Filosofía y Educación, 4, 1-16. http://revistas.uncuyo.edu.ar/ojs/index.php/saberesypracticas/article/view/2197

Valdivielso, S., Ayuste, A., Rodríguez, M. C., y Vila, E. S. (2016). Educación y género en la 
formación docente en un enfoque de equidad y democracia. En I. Carrillo (Coord.), Democracia y educación en la formación docente (pp. 117-140). Universitat Central de Catalunya. https://dialnet.unirioja.es/servlet/articulo?codigo=5783797

Velázquez-Barragán, E. (2010). La importancia de la organización escolar para el desarrollo de escuelas inclusivas (Tesis de doctorado). Universidad de Salamanca.

Villach, J. R., y Llanos, M., N. (2017). Ayudar a autorregular el aprendizaje en una situación de evaluación. Electronic Journal of Research in Education Psychology, 5(13), 805-824. http://dx.doi.org/10.25115/ejrep.v5i13.1239

Wee, E. L. (2011). Principals 'Communication Style and Parents' Involvement in School (Tesis de doctorado). University of Southampton. 